\title{
reciwlunds
}

Revista Científica Mundo de la Investigación y el Conocimiento

Ivonne Roxanna Chong Delgado a ; Holanda Mariola Merchan Ponce ${ }^{\text {b; }}$ Ángel Leonardo Pin Pin ${ }^{c}$; Pablo Cristobal Victores Pincay ${ }^{d}$

Diabetes mellitus, factores de riesgo y medidas preventivas en adolescentes del cantón Jipijapa

Diabetes mellitus, risk factors and preventive measures in adolescents of the Jipijapa canton

Revista Científica Mundo de la Investigación y el Conocimiento. Vol. 3 núm.3, septiembre, ISSN: 2588-073X, 2019, pp. 1140-1154

DOI: 10.26820/recimundo/3.(3).septiembre.2019.1140-1154

URL: http://recimundo.com/index.php/es/article/view/563

Código UNESCO: 3205 Medicina Interna

Tipo de Investigación: Artículo de Revisión

Editorial Saberes del Conocimiento

Recibido: $15 / 05 / 2019$

Aceptado: 23/06/2019

Publicado: 30/09/2019

Correspondencia: ivonne.chong@unesum.edu.ec

a. Magister en Administración de Empresas; Contador Público; Economista; Universidad Estatal del Sur de Manabí, Jipijapa; ivonne.chong@unesum.edu.ec

b. Diploma Superior en Salud Familiar y Comunitaria; Doctora en Medicina y Cirugía; Universidad Estatal del Sur de Manabí, Jipijapa; merchan.holanda@unesum.edu.ec

c. Magister en Educación Informática; Ingeniero en Computación y Redes; Universidad Estatal del Sur de Manabí, Jipijapa; angel.pim@unesum.edu.ec

d. Médico Cirujano; Hospital General del Jipijapa, Jipijapa; pablo.victores@ hgj.gob.ec 


\section{Diabetes mellitus, factores de riesgo y medidas preventivas en adolescentes del cantón Jipijapa}

Vol. 3, núm. 3., (2019)

Ivonne Roxanna Chong Delgado; Holanda Mariola Merchan Ponce; Ángel Leonardo Pin Pin;

Pablo Cristobal Victores Pincay

\section{RESUMEN}

La Universidad Estatal del Sur de Manabí viene desarrollando actividades tendientes a mantener una vinculación con la comunidad. El presente trabajo es el resultado de un proceso que se realizó con los responsables de los barrios de las diferentes parroquias urbanas del cantón Jipijapa, después de haber realizado el diagnóstico y difusión como resultados que las personas pueden presentar índices elevados de glucosa, como ayuda diagnostica de la Diabetes, debido a enfermedades y factores como hipertensión, sedentarismo, obesidad, genéticos y el uso y abuso de alcohol, drogas y carbohidratos. Del diagnóstico ejecutado en los barrios, se desprende el desconocimiento de lo que es la diabetes, afectando de manera directa a los habitantes de estos importantes sectores, ya que, no cuentan con los recursos económicos para obtener alimentos que ayudan a mejorar en la dieta diaria, al contrario se alimentan de comidas altas en grasas, azucares y carbohidratos. Por lo tanto se va a determinar los niveles de glucosa como ayuda diagnostica de la diabetes en habitantes de edad escolar de las diferentes parroquias urbanas del cantón jipijapa; las mismas que está predispuesta a recibir todo el apoyo necesario y pertinente a fin de ser los beneficiados en cuanto al proceso de capacitación en normas de alimentación, cuidados y vida saludable. Al finalizar el programa los pacientes beneficiados recibirán un diagnóstico de laboratorio en cuanto a niveles de glucosa, así mismo recibirán tratamiento en base a los resultados obtenidos por medio de las instituciones de salud que se encuentran en el territorio y la ejecución de un plan preventivo para ayudar a mejorar los niveles de glucosa y contribuir en ayudar a mejorar el estilo de vida saludable, ejecutado y aplicado por los estudiantes de las Carreras de laboratorio Clínico y Enfermería.

Palabras Claves: Diabetes; Diabetes Mellitos; Glucosa; Diagnostico; Control. 


\title{
Diabetes mellitus, factores de riesgo y medidas preventivas en adolescentes del cantón Jipijapa
}

Vol. 3, núm. 3., (2019)

Ivonne Roxanna Chong Delgado; Holanda Mariola Merchan Ponce; Ángel Leonardo Pin Pin; Pablo Cristobal Victores Pincay

\begin{abstract}
The State University of the South of Manabí comes to carry out activities aimed at maintaining a relationship with the community. The present work is the result of a process that was carried out with those responsible for the neighborhoods of the different urban parishes of the Jipijapa canton, after having made the diagnosis and dissemination as results that people can present glucose increase indices, as an aid Diagnosis of diabetes, due to diseases and factors such as hypertension, sedentary lifestyle, obesity, genetics and the use and abuse of alcohol, drugs and carbohydrates. From the diagnosis carried out in the neighborhoods, disregard the ignorance of what diabetes is, directly affecting the inhabitants of these important sectors, since they do not have the financial resources to obtain food that helps to improve in the daily diet, on the contrary they feed on foods high in fats, sugars and carbohydrates. Therefore, glucose levels will be determined as a diagnostic aid for diabetes in school-age inhabitants of the different urban parishes of the Jipijapa canton; they are predisposed to receive all necessary and relevant support in order to be the beneficiaries in the process of training in standards of food, care and healthy living. At the end of the program, the beneficiary patients will receive a laboratory diagnosis in terms of glucose levels, and they will also receive the treatment based on the results obtained through the health institutions located in the territory and the execution of a plan preventive to help improve glucose levels and contribute to help improve the healthy lifestyle, executed and applied by the students of the Clinical and Nursing Laboratory Careers.
\end{abstract}

Key Words: Diabetes; Diabetes Twins; Glucose; Diagnosis; Control. 


\section{Diabetes mellitus, factores de riesgo y medidas preventivas en adolescentes del cantón Jipijapa}

Vol. 3, núm. 3., (2019)

Ivonne Roxanna Chong Delgado; Holanda Mariola Merchan Ponce; Ángel Leonardo Pin Pin;

Pablo Cristobal Victores Pincay

\section{Introducción.}

La Diabetes es un problema de salud pública mundial que no tiene una manifestación única, sino un síndrome dentro del cual deben individualizarse diferentes entidades nosológicas. El nexo común de todas ellas es la hiperglucemia y sus consecuencias, es decir, las complicaciones específicas de hiperglicemia, las cuales son comunes a todas las formas de diabetes.

La OMS ha clasificado a la diabetes Mellitus en dos tipos: Diabetes Mellitus Tipo I y Diabetes Mellitus Tipo II, apareciendo esta última después de la cuarta década de la vida y no va asociada a destrucción autoinmune de las células beta. Sin embargo, este tipo de diabetes (tipo I) también puede aparecer en edad joven y se denomina diabetes tipo MODY (maturity-onset type diabetes of the young). (OMS, 2017)

Además de otros tipos de diabetes. La diabetes de tipo II o no insulinodependiente (NIDDM) es la forma más común y se calcula que afecta aproximadamente a un 5\% de la población general, con un componente genético importante. De hecho, se ha estimado que la concordancia en gemelos monocigóticos es del 100\% si la enfermedad comienza después de los 45 años de edad. (Leonor, Tim, \& Jessica, 2013)

La Organización Mundial de la Salud (OMS) calcula que en el mundo hay más de 220 millones de personas con diabetes. Casi el $80 \%$ de las muertes por esta enfermedad se produce en países de ingresos bajos o medios. En Ecuador, los casos notificados para diabetes Mellitus (diabetes 2) fueron de 92 629, en 2010. Sin embargo, el número es mucho mayor porque más de 


\section{Diabetes mellitus, factores de riesgo y medidas preventivas en adolescentes del cantón Jipijapa}

Vol. 3, núm. 3., (2019)

Ivonne Roxanna Chong Delgado; Holanda Mariola Merchan Ponce; Ángel Leonardo Pin Pin; Pablo Cristobal Victores Pincay

la mitad de las personas que la padecen no lo sabe. A ello hay que sumar los enfermos de diabetes 1 . Según algunos datos, en el Ecuador hay alrededor de 500 mil personas que sufren de diabetes, pero apenas unas 100 mil reciben tratamiento adecuado. Según datos entregados por el Instituto Nacional de Estadísticas y Censos (INEC, 2010), en Ecuador 4.017 personas con diabetes fallecieron, cabe recordar que esta es la segunda causa de muerte general en el país.

Existen una serie de factores de riesgos relacionados con el desarrollo de la diabetes mellitus tipo II tales como: Edad (>40 años), sexo (Femenino), raza (negra), Obesidad, Hipertensión arterial, factores genéticos (alteración de las células beta), sedentarismo, etc. por lo que cada día se hace necesario realizar estudios que nos permitan identificar nuevos factores de riesgo y a su vez obtener la prevalencia general de cada uno de ellos en nuestra población para así poder modificar dichos factores de riesgos.

En Ecuador, el incremento en la prevalencia de esta enfermedad relacionado con la edad se refleja con afectación a más de 10 por ciento de la población de ese grupo. Los resultados sobre niveles elevados de glucosa en la provincia y particularmente en el cantón de Jipijapa son alarmantes. (Luisa, y otros, 2017)

Por tal motivo y ante la falta de prevención y conocimientos de la enfermedad por parte de los habitantes de la parroquia La América, sobre la problemática que acarrea la presencia de esta patología, La Universidad Estatal Del Sur De Manabí, a través, de los estudiantes de Laboratorio Clínico y Enfermería han desarrollado un programa de vinculación con la comunidad que esta direccionado a mejorar la calidad de vida de los adultos de este sector. 


\section{Diabetes mellitus, factores de riesgo y medidas preventivas en adolescentes del cantón Jipijapa}

Vol. 3, núm. 3., (2019)

Ivonne Roxanna Chong Delgado; Holanda Mariola Merchan Ponce; Ángel Leonardo Pin Pin;

Pablo Cristobal Victores Pincay

Dada la magnitud del problema de la diabetes y sus consecuencias es importante obtener datos actuales sobre la prevalencia de la enfermedad, ya que, no existe información reciente que refleje el estado actual de los diabéticos de las parroquias Urbanas del cantón Jipijapa.

\section{Métodos y materiales}

Teniendo presente la situación problemática y la motivación para llevar a cabo el proyecto, se debe aplicar la siguiente metodología:

\section{Diagnostico}

De acuerdo al censo de población y vivienda realizado en el 2010, la parroquia tiene 3.060 habitantes, (1.638 hombres y 1.422 mujeres), con una proyección de crecimiento anual de 0,59\%, por lo que la parroquia al 2015 tiene una población de 3.151 habitantes (1.672 y 1.479 mujeres) reportado por la (INEC, 2010)

El (PDOT, 2015 ) del Cantón Jipijapa, menciona que existen algunos problemas de salud, debido a que el consumo de alimentos es muy bajo y lo hace más vulnerable a padecer de alguna patología, sumado a este mal habito alimenticio, sedentarismo y obesidad, el problema de salud se acrecienta, ya que, estas condiciones forman un conjunto de factores de riesgo de los habitantes del cantón para padecer diabetes; encontrando que la diabetes está situada en el tercer lugar de las enfermedades más comunes que se presentan en el cantón, con un total de mortalidad del 34\%, según el Censo 2010 del cantón Jipijapa. 


\section{Diabetes mellitus, factores de riesgo y medidas preventivas en adolescentes del cantón Jipijapa}

Vol. 3, núm. 3., (2019)

Ivonne Roxanna Chong Delgado; Holanda Mariola Merchan Ponce; Ángel Leonardo Pin Pin; Pablo Cristobal Victores Pincay

Una vez determinada la problemática y analizando que no existen políticas claras sobre este tema en el cantón, surge la realización de este trabajo de investigación que busca de esta manera reducir los casos de la enfermedad.

Identificación de actores

- Universidad Estatal del Sur de Manabí (UNESUM)

- Habitantes de las Parroquias urbanas del cantón Jipijapa

- Docentes y estudiantes de la carrera laboratorio clínico y enfermería

- Centro de salud Jipijapa y Hospital.

Planteamiento de objetivos

Identificar los factores de riesgo de la diabetes en habitantes del Cantón jipijapa

Con el trabajo de los estudiantes de la carrera de laboratorio clínico y enfermería se aplicara una ficha diagnostica a las beneficiarios del proyecto que se encuentran participando, con esta ficha diagnostica podremos determinar que pacientes se encuentran dentro de los factores de riesgo de contraer diabetes.

\section{Realizar pruebas de glucosa en habitantes del Cantón Jipijapa}

Una vez identificados las personas que van a participar en el desarrollo del proyecto, se los convocara con fecha y horario establecidos para que los estudiantes de la carrera de laboratorio clínico realicen la toma de muestra de sangre para realizar la prueba de glucosa en los laboratorios de nuestra carrera y así poder ayudar en el diagnóstico de la enfermedad. 


\section{Diabetes mellitus, factores de riesgo y medidas preventivas en adolescentes del cantón Jipijapa}

Vol. 3, núm. 3., (2019)

Ivonne Roxanna Chong Delgado; Holanda Mariola Merchan Ponce; Ángel Leonardo Pin Pin;

Pablo Cristobal Victores Pincay

Ejecutar un plan preventivo sobre factores de riesgo de la diabetes mellitus, correctos hábitos alimenticios, adecuada alimentación y estilo de vida para mejorar el estado del paciente

Una vez que se han tabulado, reportado y entregado los resultados de las pruebas de laboratorios por parte de los estudiantes de la carrera de laboratorio clínico y enfermería a los beneficiarios del proyecto, se acordaran visitas en cada una de las diferentes comunidades para educar a la población y para darles a conocer de qué manera pueden prevenir la diabetes, y a su vez buscar tratamientos este tipo de patologías.

Planificación de acciones a seguir

Recursos humanos

$\checkmark$ B.Q.F Karina Merchán Villafuerte Mg.- Directora Proyecto - Carrera Laboratorio Clínico

$\checkmark$ Dra. Nathaly Villafuerte Moreno- Responsable del proyecto - Carrera Laboratorio Clínico

$\checkmark$ Lcda. Yadira Patricia Ávila Piguave Mg.- Docente miembro del equipo de trabajo Carrera Enfermería

$\checkmark$ Estudiantes de las Carreras de Laboratorio Clínico y Enfermería (83).

$\checkmark$ GAD Cantón Jipijapa 


\section{Diabetes mellitus, factores de riesgo y medidas preventivas en adolescentes del cantón Jipijapa}

Vol. 3, núm. 3., (2019)

Ivonne Roxanna Chong Delgado; Holanda Mariola Merchan Ponce; Ángel Leonardo Pin Pin; Pablo Cristobal Victores Pincay

Recursos financieros

Universidad Estatal del Sur de Manabí

Investigaciones a introducir

Artículo científico

Organización y control del trabajo

Trabajo monitoreado por el B.Q.F Karina Merchán Villafuerte Mg - Directora del proyecto, Docentes responsables carrera de Laboratorio Clínico y Enfermería y técnico del área de proyectos de vinculación.

Medición de impacto

Según la población y los directivos de las Parroquias Urbanas del cantón Jipijapa mencionan que existen problemas de salud, debido a que no hay estudios realizados en cuanto a padecer patología como es Diabetes, sumado a esto las enfermedades que serían una de las causantes de esta enfermedad, el problema de salud se acrecienta, ya que, la carencia de servicios básicos y la baja condición económicas forman un conjuntos de factores peligrosos que arremeten con la vida de la comunidad; encontrando que la obesidad, hipertensión y sedentarismo y con ella las predisposiciones genéticas son factores de riesgo para contraer la diabetes. Está situada en el tercer lugar de las enfermedades más comunes que se presentan en el cantón Jipijapa con un total de mortalidad del 34\%, según el censo del 2010 del cantón. 


\section{Diabetes mellitus, factores de riesgo y medidas preventivas en adolescentes del cantón Jipijapa}

Vol. 3, núm. 3., (2019)

Ivonne Roxanna Chong Delgado; Holanda Mariola Merchan Ponce; Ángel Leonardo Pin Pin;

Pablo Cristobal Victores Pincay

\section{Resultados.}

La incidencia de la diabetes Tipo 1 es de 10 a 12 casos nuevos por cada 100.000 habitantes. De ellos aproximadamente 9 tienen menos de 15 años. Para los menores de 14 años esta incidencia es del 0,092\%, mientras que la incidencia de la diabetes Tipo 2 es de 60 a 150 casos nuevos por 100.000 habitantes (Martín, 2005).

Mediante los datos obtenidos en las parroquias rurales del cantón Jipijapa, en conjunto con sus habitantes se pudo evidenciar que la problemática principal es el desorden alimenticio y la calidad de vida que los adultos mayores tienen para desarrollar la enfermedad, además se asocia con otros factores como pueden ser la obesidad, el sedentarismo, predisposición genética y otros malos hábitos como fumar y tomar bebidas alcohólicas.

Es por este motivo que la Universidad Estatal del sur de Manabí con sus carreras de Laboratorio Clínico y Enfermería dio pie a la ejecución de este trabajo de investigación con el fin de poder mejorar la calidad de vida de estos habitantes.

Una vez establecido el problema y estimado el déficit o demanda insatisfecha, se identificó la población afectada, desagregada por sexo, nacionalidades, pueblos y edad, e indicó cuál fue su población objetivo; es decir aquella población que sería atendida en la investigación.

La población objetivo del proyecto se conformó por la demanda insatisfecha expresada en el cuadro anterior. 


\section{Diabetes mellitus, factores de riesgo y medidas preventivas en adolescentes del cantón Jipijapa}

Vol. 3, núm. 3., (2019)

Ivonne Roxanna Chong Delgado; Holanda Mariola Merchan Ponce; Ángel Leonardo Pin Pin; Pablo Cristobal Victores Pincay

\section{Cuadro $\mathbf{N}^{\circ}$ 1. Población objetivo}

\begin{tabular}{|c|c|}
\hline AÑO & POBLACIÓN OBJETIVO \\
\hline 2015 & 300 \\
\hline 2016 & 304 \\
\hline 2017 & 309 \\
2018 & 317 \\
\hline 2019 & 326 \\
\hline
\end{tabular}

La población objetivo conformada por caficultores de los cantones Jipijapa, Paján y Puerto López, se caracteriza por ser una población de escasos recursos económicos, en su gran totalidad se dedica a las actividades agropecuarias y comerciales del sector; en muchos casos de las familias poseen viviendas de construcción mixta con deficiente abastecimiento de servicios básicos como: alcantarillado y agua potable.

El autor (Medline Plus, 2014) expresa que para las pruebas y exámenes se puede utilizar un análisis de orina para buscar hiperglucemia; sin embargo, una prueba de orina sola no diagnostica diabetes. El médico puede sospechar que usted tiene diabetes si su azúcar en la sangre es superior a $200 \mathrm{mg} / \mathrm{dL}$. Para confirmar el diagnóstico, se deben hacer uno o más de los siguientes exámenes:

Exámenes de sangre:

- Glucemia en ayunas: se diagnostica diabetes si el resultado es mayor de $126 \mathrm{mg} / \mathrm{dL}$ en dos oportunidades. Los niveles entre 100 y $126 \mathrm{mg} / \mathrm{dL}$ se denominan alteración de la glucosa en ayunas o prediabetes. Dichos niveles se consideran factores de riesgo para la diabetes tipo 2 . 


\section{Diabetes mellitus, factores de riesgo y medidas preventivas en adolescentes del cantón Jipijapa}

Vol. 3, núm. 3., (2019)

Ivonne Roxanna Chong Delgado; Holanda Mariola Merchan Ponce; Ángel Leonardo Pin Pin;

Pablo Cristobal Victores Pincay

- Examen de hemoglobina A1c:

Normal: menos de $5.7 \%$

Prediabetes: entre $5.7 \%$ y $6.4 \%$

Diabetes: $6.5 \%$ o superior.

- Prueba de tolerancia a la glucosa oral: Se diagnostica diabetes si el nivel de glucosa es superior a $200 \mathrm{mg} / \mathrm{dL}$ luego de 2 horas (esta prueba se usa con mayor frecuencia para la diabetes tipo 2).

Las pruebas de detección para diabetes tipo 2 en personas asintomáticas se recomiendan para:

- Niños obesos que tengan otros factores de riesgo para diabetes, comenzando a la edad de 10 años y repitiendo cada dos años.

- Adultos con sobrepeso (IMC superior a 25) que tengan otros factores de riesgo.

- Adultos de más de 45 años, repitiendo cada tres años.

Sin embargo, durante la investigación las pruebas realizadas fue la de los niveles de glucosa en sangre y su relación con Diabetes Mellitus tipo II. Se les realizaron los exámenes de laboratorio a 400 adolescentes que corresponde al $100 \%$ de la población en estudio de las parroquias urbanas del Cantón Jipijapa. Al tercer mes de ejecución, se realiza el levantamiento de la información diagnóstica a los 400 adolescentes, lo que permitió identificar los factores de riesgo de la diabetes. Sin embargo, al finalizar el tercer mes se aplicó la ficha diagnostica a los 


\section{Diabetes mellitus, factores de riesgo y medidas preventivas en adolescentes del cantón Jipijapa}

Vol. 3, núm. 3., (2019)

Ivonne Roxanna Chong Delgado; Holanda Mariola Merchan Ponce; Ángel Leonardo Pin Pin; Pablo Cristobal Victores Pincay

400 adolecentes tomado los indicadores antropométricos.

Durante la determinación de los niveles de glucosa y su relación con la Diabetes Mellitus tipo II se observó que más del 80\% presentó niveles de glucosa elevados en sangre por lo que, los estudiantes de la carrera de laboratorio clínico y Enfermería difunden el plan preventivo en los adolescentes que presentaron niveles elevados de glucosa. Los temas abordados mediante el plan preventivo fueron los siguientes: factores de riesgo de la Diabetes Mellitus, correctos hábitos alimenticios y estilo de vida saludable logrando disminuir los niveles de glucosa considerablemente.

Mediante la aplicación del plan preventivo se logra disminuir considerablemente los niveles de glucosa en sangre de los participantes, de acuerdo a los seguimientos de exámenes de laboratorios aplicados meses posteriores a la aplicación del plan lo que determinó que la investigación logro mejorar la calidad de vida de los habitantes de este Cantón a pesar de las carencias económicas e informativas que poseen acerca de esta patología.

\section{Conclusiones.}

Durante el desarrollo de la investigación se observó que la Diabetes Mellitus es un tipo de enfermedad que amenaza cada día con más frecuencia a la población mundial. Tomar razón acerca de los cuidados para prevenir esta enfermedad, está de parte de las entidades públicas a fin de incorporar campañas informativas que brinden información a la población y los ayude a concientizar acerca de este tema. 


\section{Diabetes mellitus, factores de riesgo y medidas preventivas en adolescentes del cantón Jipijapa}

Vol. 3, núm. 3., (2019)

Ivonne Roxanna Chong Delgado; Holanda Mariola Merchan Ponce; Ángel Leonardo Pin Pin;

Pablo Cristobal Victores Pincay

Aunado a esto, en la investigación realizada en el Cantón Jipijapa se observó que la población adolescente estudiada presentó niveles elevados de glucosa lo que causas ruido ante una problemática que cada día se hace mayor y que debido a esto se toma la decisión de implementar un plan preventivo el cual se observa que sirvió de gran ayuda a los adolescentes que presentaron altos niveles de glucosa, ya que, estos niveles se lograron bajar considerablemente.

Lo más alarmantes de todo es que esta patología se está presente en la población joven, por lo que, la implementación del plan preventivo se debe continuar así como también la evaluación de los exámenes de laboratorio para mejorar la calidad de vida de las personas que habitan en el Cantón Jipijapa.

Este tipo de proyectos o planes de prevención se recomiendan realizar en los diferentes Cantones del Ecuador a modo de crear conciencia en la población joven y adulta para así disminuir los de riesgos en el padecimiento de esta patología que con los años, y de no ser tratada puede llegar a ser mortal.

Finalmente se puede concluir, que los factores de riesgo más relevantes para la presencia de esta enfermedad son el consumos de comidas grasas, azucares y carbohidratos en excesos que alteran los niveles de glucosa en la sangre así como también la toma excesiva de bebidas alcohólicas y llevar una vida sedentaria.

\section{Bibliografía.}

INEC. (2010). Instituto Nacional de Estadísticas y Censos. Ecuador: INEC. 


\section{Diabetes mellitus, factores de riesgo y medidas preventivas en adolescentes del cantón Jipijapa}

Vol. 3, núm. 3., (2019)

Ivonne Roxanna Chong Delgado; Holanda Mariola Merchan Ponce; Ángel Leonardo Pin Pin; Pablo Cristobal Victores Pincay

Leonor, G., Tim, N., \& Jessica, B. (2013). ATLAS de la DIABETES de la FID. $6^{\circ}$ Edicion.

Luisa, C., María, V., Gabriela, C., Robert, Á., Roberto, A., Joselyn, R., \& Valmore, B. (2017). Prevalencia de la diabetes mellitus tipo 2 y sus factores de riesgo en individuos adultosEcuador. Avances en Biomedicina , 20-54.

Martín. (2005). Diabetes Mellitus. 23-34.

OMS. (2017). Informe mundial sobre la diabetes. Organizacion Mundial de la Salud.

PDOT. (2015 ). PLAN DE DESARROLLO Y ORDENAMIENTO TERRITORIAL. Ecuador. 